EESTI NSV TEADUSTE AKADEEMIA TOIMETISED.

FUOSIKA - MATEMAATIKA

ИЗВЕСТИЯ АКАДЕМИИ НАУК ЭСТОНСКОИ ССР. ФИЗИКА * МАТЕМАТИКА

PROCEEDINGS OF THE ACADEMY OF SCIENCES OF THE ESTONIAN SSR. PHYSICS - MATHEMATICS

$1986,35,1$

удК 621.314 .63

T. TOMСOH

\title{
ИДЕАЛИЗИРОВАННАЯ СИСТЕМА ВЫПРЯМИТЕЛЕЙ НА ОБЩИХ ТИРИСТОРАХ
}

\author{
(Представил И. Эпик)
}

\section{Введение}

B $\left[{ }^{1,2}\right]$ отмечена возможность построения системы электропитания гальванически объединенных нагрузок на базе выпрямителей с группой общих тиристоров (рис. 1). В настоящей статье проведен анализ аналогичной идеализированной системы. Идеализация системы позволяет выделить основные электромагнитные процессы и указать принципиальные ограничения, непреодолимые усовершенствованием технической реализации. Целью анализа является определение регулировочных характеристик, диапазона работы и иных ограничений, которые следует учесть при разработке системы выпрямителей.

При анализе приняты следующие упрощающие предпосылки:

1) вентили являются идеальными полууправляемыми ключами;

2) коммутация вентилей - мгновенная;

3) питающая сеть симметрична и синусоидальна;

4) постоянная времени индуктивно-активной нагрузки стремится к бесконечности;

5) система стабилизации тока разомкнута.

Для краткости изложения назовем автономную тиристорную группу $V_{1}^{\prime}, V^{\prime}{ }_{3}, V^{\prime}{ }_{5}$ первым регулятором, на который воздействует управляющий сигнал системы импульсно-фазового управления (СИФУ) с фазой $\boldsymbol{\alpha}^{\prime}$. Соответственно автономная тиристорная группа $V_{1}^{\prime \prime}, V_{3}^{\prime \prime}, V_{5}^{\prime \prime}$ называется вторым регулятором, управляющий сигнал которого имеет фазу $\boldsymbol{\alpha}^{\prime \prime}$. Примем, что всерда $\alpha^{\prime \prime} \geqslant \alpha^{\prime}$, т. е. первый регулятор является опережающим (ведущим), второй регулятор - запаздывающим (ведомым).

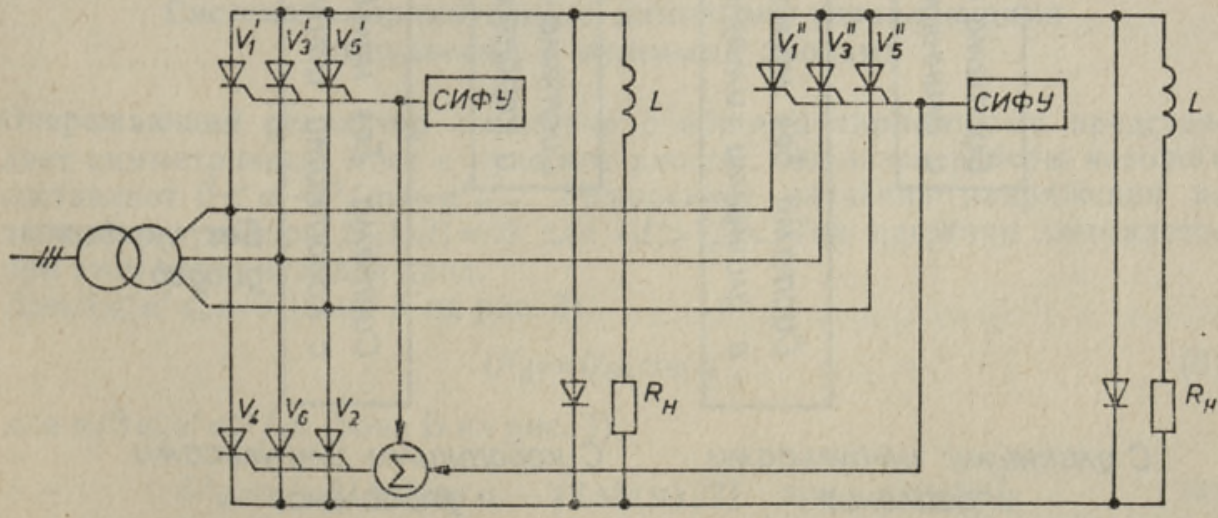

Pис. 1. 
Ограничим число входящих в систему регуляторов на два, что есть минимальное необходимое и достаточное число для образования системы. Всякая большая система может быть рассмотрена по парам: некоторый выделенный регулятор и все остальные регуляторы в совокупности, системные свойства которой задаются опережающим в этом числе регулятором.

Опережающий регулятор совместно с общей группой тиристоров образует нормальный 6-пульсный мост. Запаздывающий регулятор совместно с общей группой тиристоров образует несимметричный мост. При условии управления током до нуля (что, очевидно, требуется) возникает необходимость селективного отключения запаздывающего регулятора. При этом следует учесть, что запирать общие тиристоры нельзя, а снятие управляющих импульсов запаздывающего регулятора приводит лишь к аварийным токам. Поэтому селективное отключение нагрузок в описываемой системе электропитания требует обязательного применения нулевых диодов. Характер управляющего импульса (короткий, длинный) также влияет на свойства системы. Указанные параметры позволяют классифицировать системы выпрямителей на общих тиристорах (рис. 2). Наличие нулевого диода обусловливает при $\alpha^{\prime \prime}-\alpha^{\prime}=$ $=\Delta_{\alpha}>\pi / 3$ режим прерывистого тока в регуляторе, несмотря на непрерывность тока в цепи нагрузки. Определение регулировочной характеристики $U_{d}=f(\alpha)$ в относительных единицах осуществляется по следующей методике.

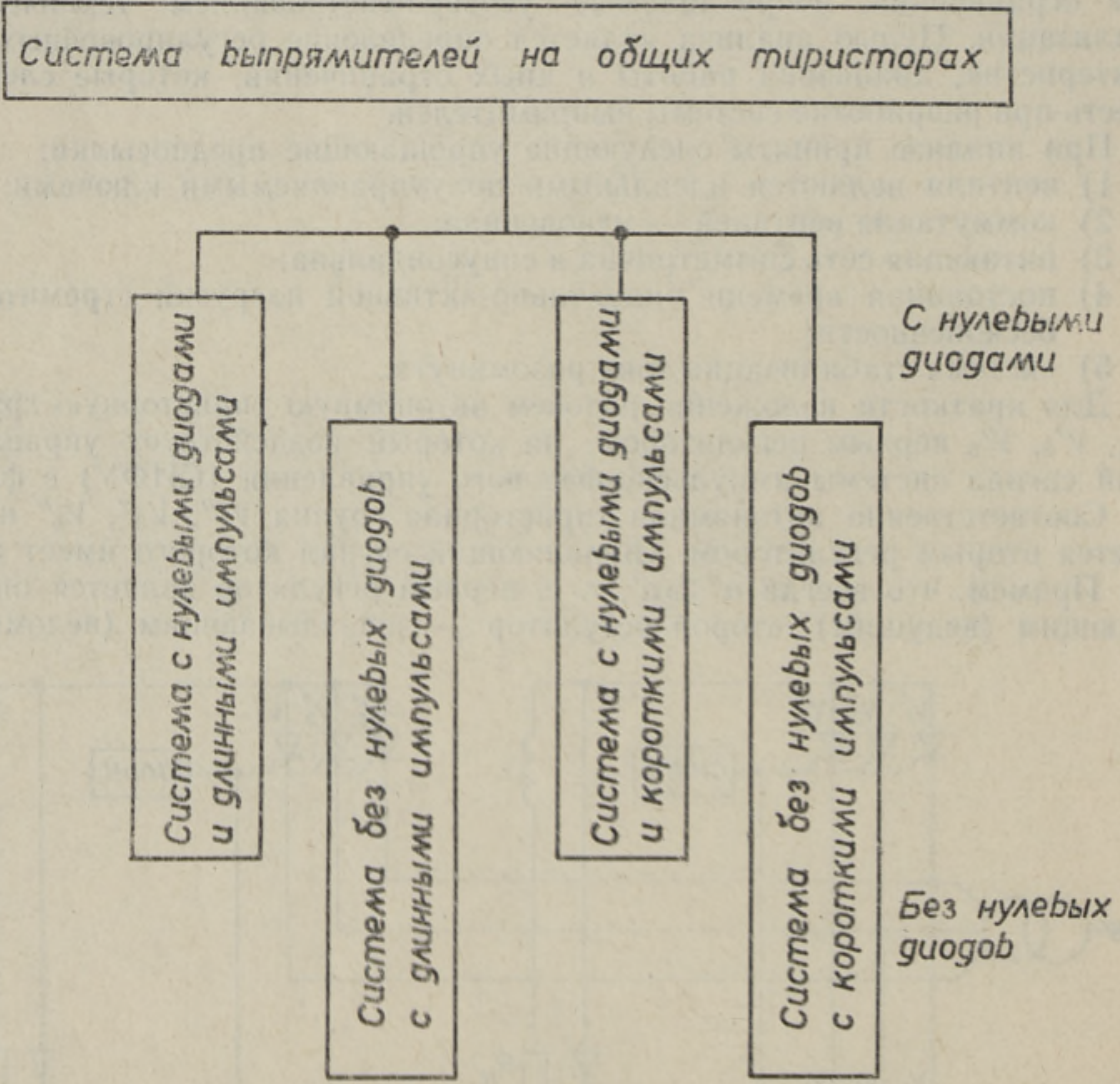

C gлинными импульсами упраьления

С корогпкими импульсами упраьления

Рис. 2. 
Пॉостоянная составляющая несимметричного моста выражается суммой интегралов

$$
U_{d}^{\prime \prime}=\frac{1}{2 \pi}\left(\sum_{i=1}^{3} \int_{\alpha_{t H}}^{\alpha_{l k}} E \sin (\omega t+\pi / 3) d \omega t+\sum_{j=1}^{3} \int_{\alpha_{f H}}^{\alpha_{l k}} E \sin \omega t d \omega t\right),
$$

где $i$ - индекс вентиля регулятора, $j$ - индекс вентиля общей группы, $E-$ амплитуда линейного напряжения, $\alpha_{i H}, \alpha_{j H}-$ нижний предел интегрирования, $\alpha_{i k}, \alpha_{j k}-$ верхний предел интегрирования. Подынтегральные синусные функции сдвинуты на $\pi / 3$ с целью применения единой угловой базы для двух последовательных вентилей. С учетом известного значения напряжения (холостого хода) неуправляемого диодного выпрямителя $U_{d 0}$ найдем по (1)

$$
U_{d}^{\prime \prime}=\frac{U_{d 0}}{2}\left(\int_{\alpha_{t H}}^{\alpha_{t k}} \sin (\omega t+\pi / 3) d \omega t+\int_{\alpha_{j H}}^{\alpha_{j k}} \sin \omega t d \omega t\right) .
$$

Значения $\alpha_{i H} \ldots \alpha_{j h}$ определены в зависимости от классификационных признаков частного регулятора и режима его работы в разных угловых зонах следующим образом.

I режим: $\alpha^{\prime}<\pi / 3, \alpha^{\prime \prime}<\pi / 3$; IIa режим: $\alpha^{\prime}<\pi / 3, \pi / 3<\alpha^{\prime \prime}<2 / 3 \pi$; IIб режим: $\alpha^{\prime}<\pi / 3, \pi / 3<\alpha^{\prime \prime}<2 / 3 \pi$; III режим: $\alpha^{\prime}<\pi / 3,2 / 3 \pi<\alpha^{\prime \prime}<\pi$; IV режим: $\pi / 3<\alpha^{\prime}<2 / 3 \pi, \pi / 3<\alpha^{\prime \prime}<2 / 3 \pi$ Va режим: $\pi / 3<\alpha^{\prime}<2 / 3 \pi, 2 / 3 \pi<\alpha^{\prime \prime}<" 1$; Vб режим: $\pi / 3<\alpha^{\prime}<2 / 3 \pi, 2 / 3 \pi<\alpha^{\prime \prime}<\pi$; VI режим: $2 / 3 \pi<\alpha^{\prime}<\pi, 2 / 3 \pi<1 \alpha^{\prime \prime}<\pi$;

$$
\begin{array}{ll}
\text { всегда } & \Delta_{\alpha}=\alpha^{\prime \prime}-\alpha^{\prime}<\pi / 3 . \\
& \Delta_{\alpha}<\pi / 3 . \\
& \Delta_{\alpha}>\pi / 3 . \\
\text { всегда } & \Delta_{\alpha}>\pi / 3 . \\
\text { всегда } & \Delta_{\alpha}<\pi / 3 . \\
& \Delta_{\alpha}<\pi / 3 . \\
& \Delta_{\alpha}>\pi / 3 . \\
\text { всегда } & \Delta_{\alpha}<\pi / 3 .
\end{array}
$$

Отметим, что VI режим работы может существовать лишь в регулятоpax без нулевых диодов. Несмотря на большое количество режимов работы и вариантов частных классификационных признаков, множество интегральных выражений по (2) сокращается ввиду совпадения пределов интегрирования. Для сокращения объема статьи ниже приводятся лишь уточняющие начальные условия и конечные выражения регулировочных характеристик. Совпадающие функции отличающихся переменных объединены, например, $U_{d}^{\prime}=f(\alpha)$ при $\alpha \in\left\{\alpha^{\prime}\right\}, U_{d}^{\prime \prime}=f(\alpha)-$ по той же формуле при $\alpha \in\left\{\alpha^{\prime \prime}\right\}$.

\section{Система выпрямителей с длинными управляющими импульсами и нулевыми диодами}

Опережающий регулятор совместно с общими тиристорами представляет симметричный мост с нулевым диодом, диапазон работы которого составляет $0<\alpha^{\prime}<^{2} / 3 \pi=\alpha_{\max }^{\prime}$. Мгновенное значение напряжения на выходе регулятора $U_{d}(\omega t)=0$ для $\omega t>2 / 3 \pi$. Ток нагрузки замыкается при этом через нулевой диод.

Для $0 \leqslant \alpha^{\prime} \leqslant \pi / 3$ (зона А на рис. 3 ) :

$$
U^{\prime}{ }_{d}=U_{d 0} \cos \alpha^{\prime},
$$

для $\pi / 3 \leqslant \alpha^{\prime} \leqslant 2 / 3 \pi$ (зона Б на рис. 3 ):

$$
U_{d}^{\prime}=U_{d 0}[1+(\cos \alpha-\sqrt{3} \sin \alpha) / 2] \text { при } \alpha \in\left\{\alpha^{\prime}\right\} .
$$

Регулировочная характеристика совпадает с соответствующей характе- 
ристикой запаздывающего регулятора при $\Delta_{\alpha}=0$ (см. кривую «нормальный мост» на рис. 4).

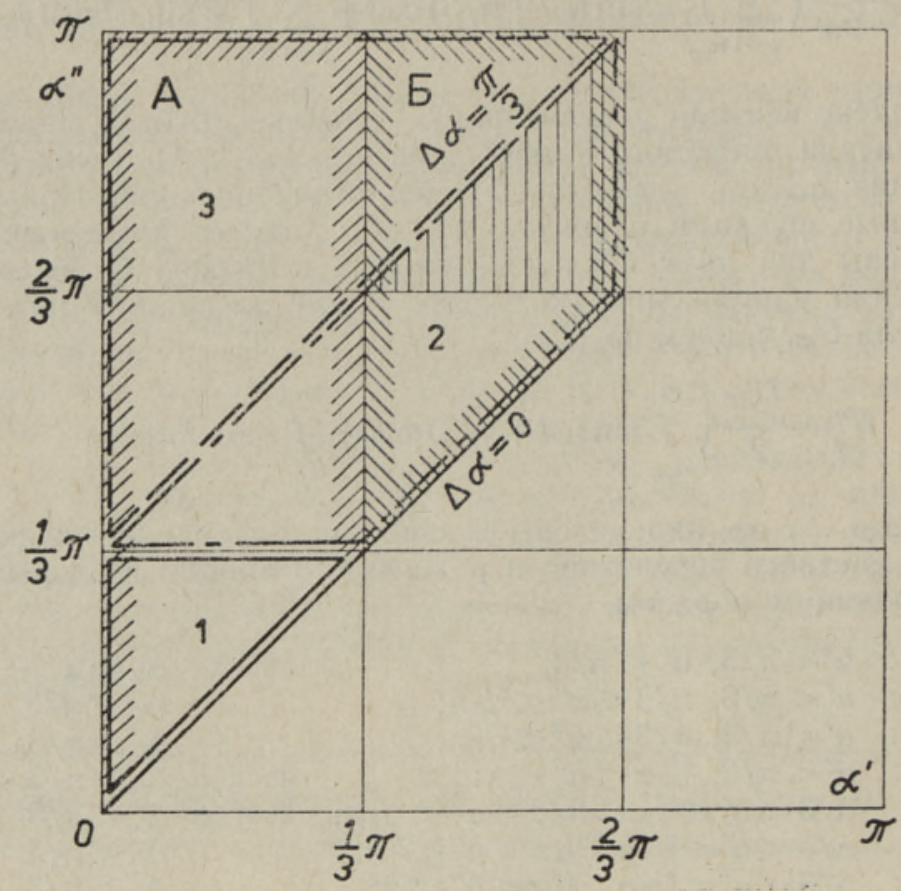

Рис. 3.

Запаздывающий регулятор. Исходные для анализа по (2) данные сосредоточены в табл. 1 и позволяют получить следующие регулировочные характеристики.

Для $\alpha^{\prime}$ и $\alpha^{\prime \prime}$, принадлежащих к зоне 1 на рис. 3 ,

$$
U_{d}^{\prime \prime}=U_{d}^{\prime} / 2+U_{d 0} \cos \alpha^{\prime \prime} / 2
$$

Для $\boldsymbol{\alpha}^{\prime}$ и $\boldsymbol{\alpha}^{\prime \prime}$, принадлежащих к зоне 2 на рис. 3 ,

$$
U_{d}^{\prime \prime}=U_{d}^{\prime} / 2+L U_{d 0}\left(1+\frac{\cos \alpha^{n}-\sqrt{3} \sin \alpha^{\prime \prime}}{2}\right) / 2,
$$

где весовая функция

$$
\begin{aligned}
& L=1, \text { если }\left(1+\frac{\cos \alpha^{\prime \prime}-\sqrt{3} \sin \alpha^{\prime \prime}}{2}\right)>0 \\
& L=0, \text { если }\left(1+\frac{\cos \alpha^{\prime \prime}-\sqrt{3} \sin \alpha^{\prime \prime}}{2}\right) \leqslant 0 .
\end{aligned}
$$

Для $\alpha^{\prime}$ и $\alpha^{\prime \prime}$, принадлежащих к зоне 3 на рис. 3

$$
U_{d}^{\prime \prime}=U_{d 0}(1+\cos \alpha) / 2 \text { при } \alpha \in\left\{\alpha^{\prime \prime}\right\} .
$$

Рассчитанные по (5)-(7) регулировочные характеристики $U_{d}^{\prime \prime}=$ $=f\left(\alpha^{\prime \prime}, \Delta_{\alpha}\right)$ и соответствующие экспериментально определенные зависимости представлены на рис. 4. Отметим следующие особенности системы: 


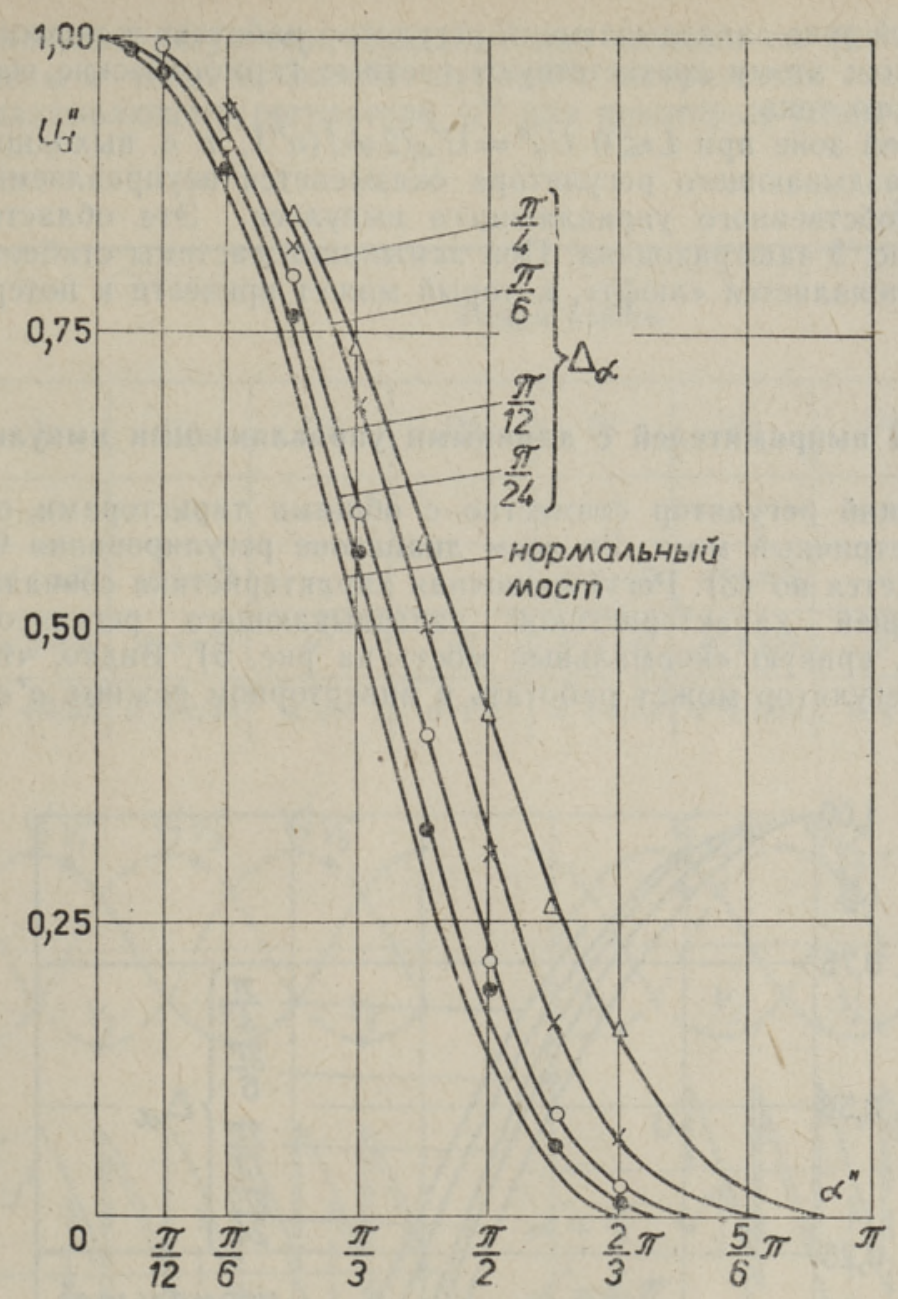

Рис. 4.

таблица 1

\begin{tabular}{|c|c|c|c|c|c|c|c|c|}
\hline \multirow{2}{*}{$\begin{array}{c}\text { Преде } \\
\text { лы ин- } \\
\text { тегри- } \\
\text { рова- } \\
\text { ния } \\
(2)\end{array}$} & \multicolumn{6}{|c|}{ Режим работы } & \multicolumn{2}{|c|}{$z$} \\
\hline & I & IIa & ІІ6 & III & IV & $\mathrm{Va}$ & V6 & VI \\
\hline$\alpha_{i H}$ & $\alpha^{\prime \prime}$ & $\alpha^{\prime \prime}$ & - & - & $\alpha^{\prime \prime}$ & - & - & - \\
\hline$\alpha_{i k}$ & $\alpha^{\prime}+\pi / 3$ & $\alpha^{\prime}+\pi / 3$ & - & - & $2 / 3 \pi$ & - & - & - \\
\hline$\alpha_{j H}$ & $\alpha^{\prime}+\pi / 3$ & $\alpha^{\prime}+\pi / 3$ & $\alpha^{\prime \prime}$ & $\alpha^{\prime \prime}$ & $\alpha^{\prime}+\pi / 3$ & $\alpha^{\prime}+\pi / 3$ & $\alpha^{\prime \prime}$ & - \\
\hline$\alpha_{j k}$ & $\alpha^{\prime \prime}+2 / 3 \pi$ & $\pi$ & $\pi$ & $\pi$ & $\pi$ & $\pi$ & $\pi$ & - \\
\hline
\end{tabular}

1) в третьей зоне запаздывающий регулятор приобретает автономность, т. е. $U_{d}^{\prime \prime} \neq f\left(\alpha^{\prime}\right)$; 
2) в третьей зоне запаздывающий регулятор работает в режиме прерывистых токов; этому соответствуют счетные гармонические составляющие входного тока;

3) во второй зоне при $L \leqslant 0 U_{d}^{\prime \prime}=U_{d}^{\prime} / 2 \neq f\left(\alpha^{\prime \prime}\right)$, т. е. выходное напряжение запаздывающего регулятора оказывается неуправляемым относительно собственного управляющего импульса. Эта область второй зоны на рис. 3 заштрихована. При замыкании системы стабилизации в этой зоне появляется «люфт», который может привести к потере устойчивости.

\section{Система выпрямителей с длинными управляющими импульсами}

Опережающий регулятор совместно с общими тиристорами представляет симметричный мост. Во всем диапазоне регулирования $0<\alpha^{n}<\pi$ $U_{d}$ выражается по (3). Регулировочная характеристика совпадает с соответствующей характеристикой запаздывающего регулятора при $\Delta_{\alpha}=0$ (см. кривую «нормальный мост» на рис. 5). Видно, что опережающий регулятор может работать в инверторном режиме $\alpha^{\prime}<\pi / 2$.

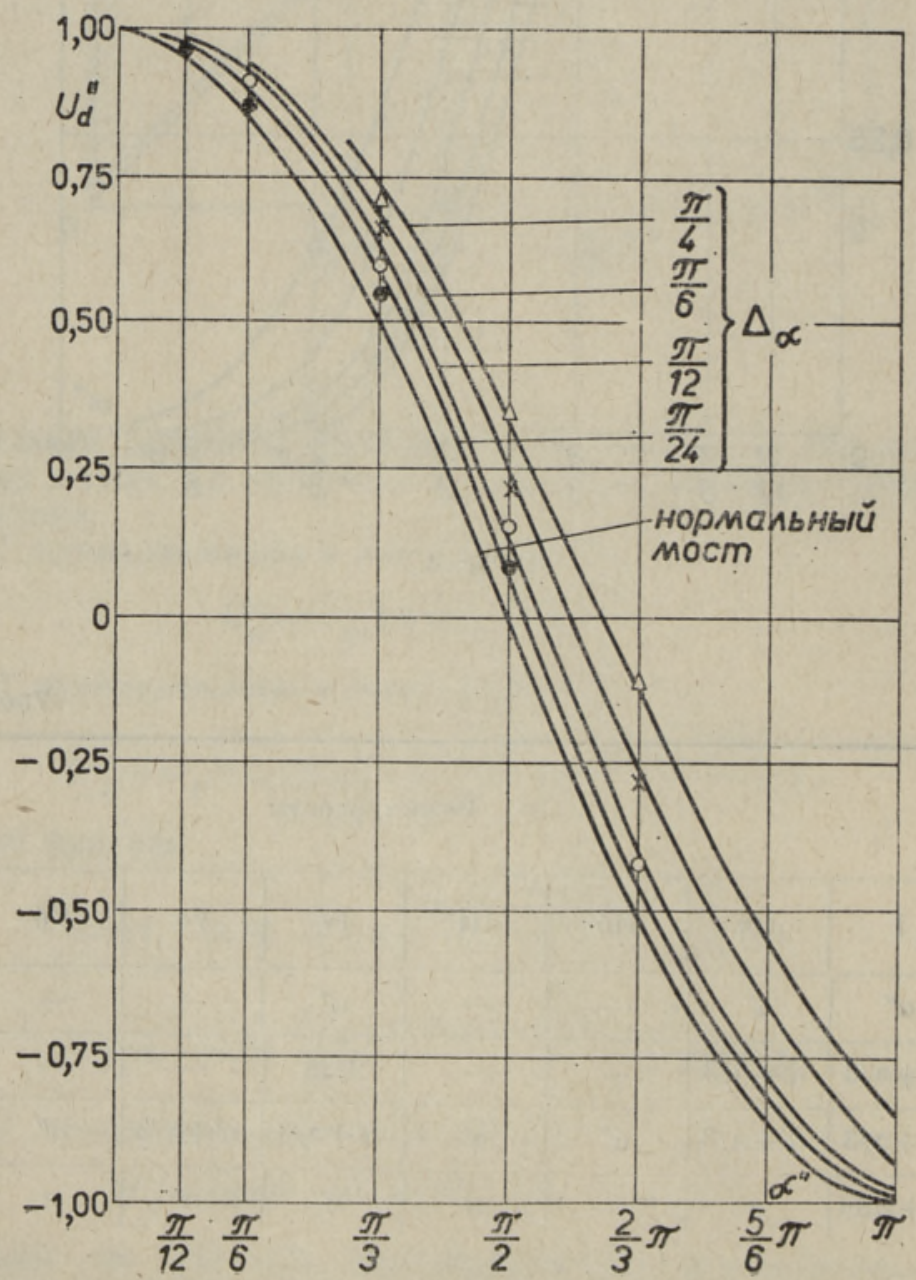

Рис. 5 , 
Запаздывающий регулятор. Исходные для анализа по (2) данные сосредоточены в табл. 2. Эпюры выходного напряжения опережающего $U^{\prime}$ и запаздывающего регулятора $U^{\prime \prime}$ для некоторых углов управления

таблица 2

\begin{tabular}{c|c|c|c|c|c|c|c|c}
\hline $\begin{array}{c}\text { Преде- } \\
\text { лы ин- } \\
\text { тегри- } \\
\text { рова- } \\
\text { ния } \\
\begin{array}{c}\text { для } \\
(2)\end{array}\end{array}$ & I & IIa & II6 & III & IV & Va & V6 & VI \\
\cline { 2 - 8 }$\alpha_{i H}$ & $\alpha^{\prime \prime}$ & $\alpha^{\prime \prime}$ & - & - & $\alpha^{\prime \prime}$ & & - & $\alpha^{\prime \prime}$ \\
\hline$\alpha_{i k}$ & $\alpha^{\prime}+\pi / 3$ & $\alpha^{\prime}+\pi / 3$ & - & - & $\alpha^{\prime}+\pi / 3$ & $\alpha^{\prime}+\pi / 3$ & - & $\alpha^{\prime}+\pi / 3$ \\
\hline$\alpha_{j H}$ & $\alpha^{\prime}+\pi / 3$ & $\alpha^{\prime}+\pi / 3$ & $\alpha^{\prime \prime}$ & $\alpha^{\prime \prime}$ & $\alpha^{\prime}+\pi / 3$ & $\alpha^{\prime}+\pi / 3$ & $\alpha^{\prime \prime}$ & $\alpha^{\prime}+\pi / 3$ \\
\hline$\alpha_{j k}$ & $\alpha^{\prime \prime}+2 / 3 \pi$ & $\alpha^{\prime \prime}+2 / 3 \pi$ & $\alpha^{\prime}+\pi$ & $\alpha^{\prime}+\pi$ & $\alpha^{\prime \prime}+2 / 3 \pi$ & $\alpha^{\prime \prime}+2 / 3 \pi$ & $\alpha^{\prime}+\pi$ & $\alpha^{\prime \prime}+2 / 3 \pi$ \\
\end{tabular}
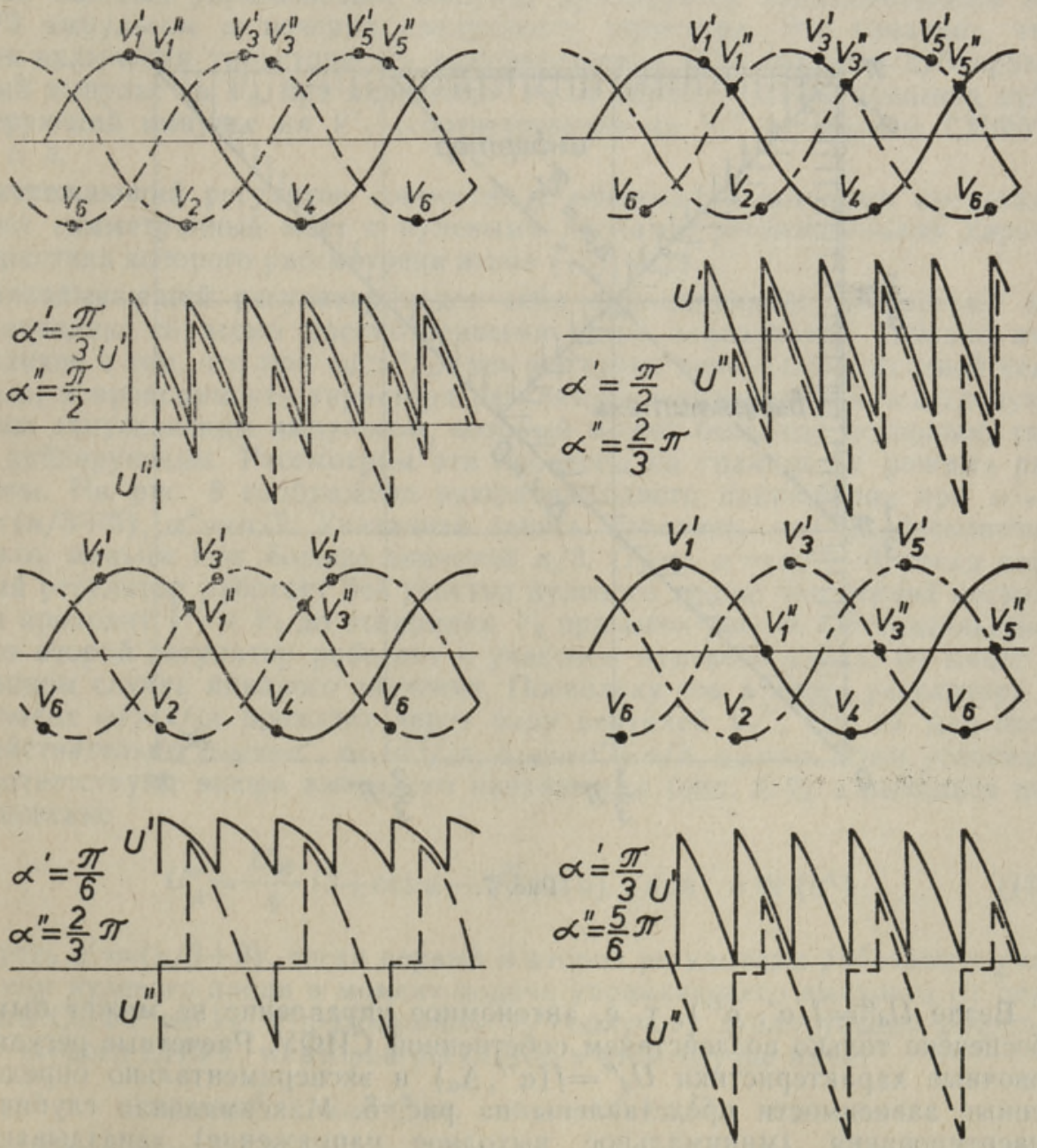

Рис. 6. 
изображены на рис. 6. Обратим внимание на то, что при $\Delta_{\alpha}>\pi / 3$ интервал проводящего состояния запаздывающего регулятора совпадает с интервалом проводимости тиристора общей группы той же фазы. Например, одновременно отперты $V_{1}^{\prime \prime}, V_{4} ; V_{3}^{\prime \prime}, V_{6}$ и $V_{5}^{\prime \prime}, V_{2}$. В указанном интервале совпадения ток нагрузки проходит два последовательно соединенных вентиля, в результате чего мгновенное значение выходного напряжения равно нулю, и за этот интервал инвертирование энергии не может быть осуществлено. Вне указанного интервала при отрицательном анодном напряжении тиристора осуществляется инвертирование энергии. Соответственно осуществляется инвертирование по интегральному значению напряжения. Это означает, что использование запаздывающего регулятора для работы в инверторном режиме вполне возможно, однако регулятор используется для инвертирования не совсем эффективно, если $\Delta_{\alpha}>\pi / 3$. Зоны работы запаздывающего регулятора приведены на рис. 7. Для всех $\alpha^{\prime}$ и $\alpha^{\prime \prime}$

$$
U_{d}^{\prime \prime}=U_{d 0}\left(\cos \alpha^{\prime}+\cos \alpha^{\prime \prime}\right) / 2 \text {. }
$$

При условии, что $\Delta_{\alpha}>\pi / 3$, осуществляется вышеупомянутый режим совпадающих проводимостей. На рис. 7 эта зона ограничена осевой линией.

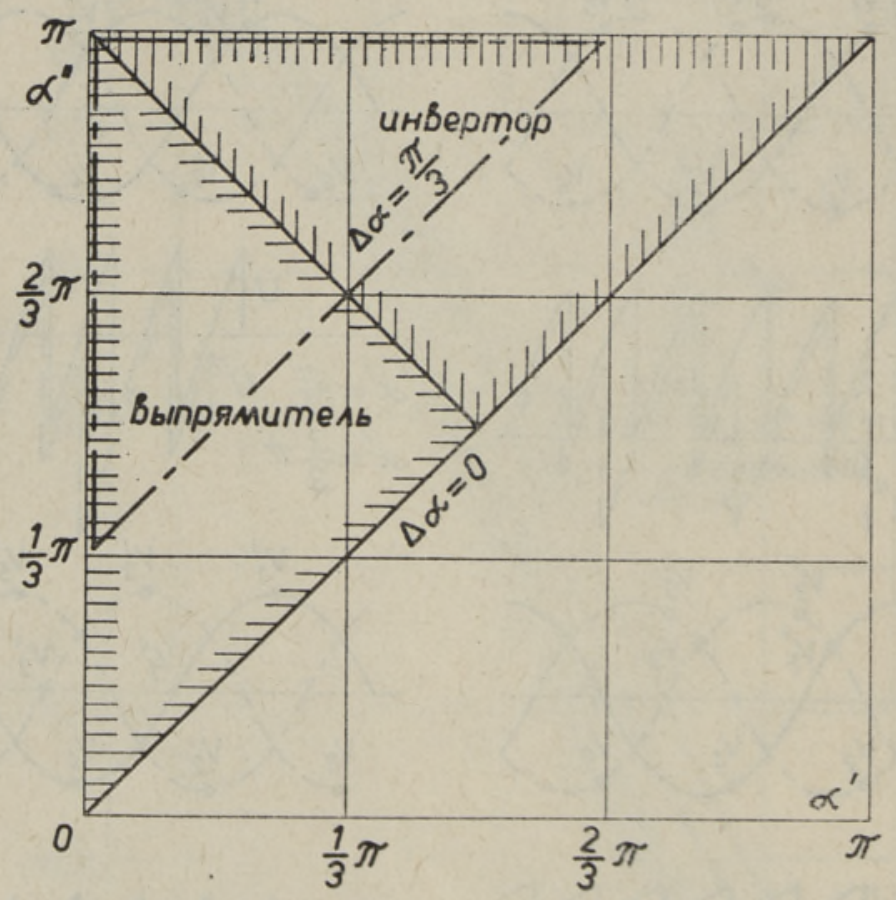

Рнс. 7.

Везде $U_{d}^{\prime \prime}=f\left(\boldsymbol{\alpha}^{\prime}, \boldsymbol{\alpha}^{\prime \prime}\right)$, т. е. автономное управление не может быть обеспечено только воздействием собственной СИФУ. Расчетные регулировочные характеристики $U_{d}^{\prime \prime}=f\left(\alpha^{\prime \prime}, \Delta_{\alpha}\right)$ и экспериментально определенные зависимости представлены на рис. 5. Максимальная глубина инвертирования (минимальное выходное напряжение) запаздывающего регулятора составляет 


$$
\min U_{d}^{\prime \prime}\left(\alpha^{\prime}\right)=U_{d 0}\left(\cos \alpha^{\prime}-1\right) / 2,
$$

что заставляет применить функционально управляемое ограничение в СИФУ запаздывающего регулятора.

\section{Система выпрямителей с короткими управляющими импульсами без нулевых диодов}

При условии, что работа регулятора может быть обеспечена одним коротким управляющим импульсом, свойства данной системы функционально тождественны системе выпрямителей с длинными управляющими импульсами без нулевых диодов (табл. 2, рис. 5-7, формулы (8), (9)) .

\section{Система выпрямителей с короткими управляющими импульсами и нулевыми диодами}

Предположим, что работа тиристоров обеспечена традиционным способом: каждый управляющий импульс дублируется запаздывающим на $\pi / 3$ импульсом включения следующего тиристора. Это означает, что при включении тиристора $V_{1}^{\prime}$ (соответственно $V_{1}^{\prime \prime}$ ) подается дублирующий импульс на $V_{6}$, при включении $V_{2}$ от первого СИФУ подается дублирующий импульс на $V_{1}^{\prime}$ (соответственно на $V_{1}^{\prime \prime}$ от второго СИФУ) и т. д.

Опережающий регулятор совместно с общими тиристорами представляет симметричный мост с нулевыми диодами, регулировочная характеристика которого рассмотрена выше $((3),(4))$.

Запаздывающий регулятор ведет себя принципиально по-другому по сравнению со всеми рассмотренными выше вариантами. Связано это отличие с тем, что при $\omega t>2 / 3 \pi$ ток нагрузки переходит в нулевой вентиль, и проводимость тиристора регулятора восстанавливается следующим запускающим импульсом, который может быть как основным, так и дублирующим. Рассмотрим эти процессы на границе IV режима работы. На рис. 8 изображена эпюра выходного напряжения при $\alpha^{\prime}=$ $=(\pi / 3 \mp 0), \alpha^{\prime \prime}=\pi / 2$. Указанная запись означает, что $\alpha^{\prime}$ бесконечно мало, меньше или больше значений $\pi / 3$. Пусть $\alpha^{\prime}=\pi / 3-0$, тогда первый регулятор работает без участия нулевого диода: ток первой нагрузки проходит $V_{1}^{\prime}$ и $V_{6}$ до отпирания $V_{2}$ при $\omega t=2 / 3 \pi-0$. Обстоятельство, что второй регулятор работает с участием нулевого диода, не имеет в данном случае никакого значения. Поскольку ток второго регулятора в момент $\omega t \approx^{2} / 3 \pi$ проходит через пару вентилей $V_{1}^{\prime \prime}, V_{2}$, то для него действительны $\alpha_{i H}=\alpha^{\prime \prime}, \alpha_{i k}=2 / 3 \pi, \alpha_{j H}=\alpha^{\prime}+\pi / 3, \alpha_{j k}=\pi$. Этим условиям соответствуют эпюра выходного напряжения (рис. 8,6$)$ и выходное напряжение

$$
U_{d}^{\prime \prime}=\frac{U_{d 0}}{4}(3+\cos \alpha-\sqrt{3} \sin \alpha) \quad \text { при } \quad \alpha \in\left\{\alpha^{\prime \prime}\right\} .
$$

Пусть $\alpha^{\prime}=(\pi / 3+0)$, тогда первый и второй регуляторы работают с участием нулевого диода в момент подачи управляющего импульса от первого СИФУ на $V_{2}, V^{\prime}{ }_{1}$. Поскольку $V_{1}{ }^{\prime \prime}$ получает дублирующий импульс от второго СИФУ, то второй регулятор в этом случае имеет

$$
\alpha_{i H}=\alpha^{\prime \prime}, \quad \alpha_{i k}=2 / 3 \pi, \quad \alpha_{j H}=\alpha^{\prime \prime}+\pi / 3, \quad \alpha_{j k}=\pi .
$$

Этим условиям соответствуют эпюра выходного напряжения на рис. 8,8 и выходное напряжение по формуле (4) при $\alpha \in\left\{\alpha^{\prime \prime}\right\}$. $U_{d}^{\prime \prime}$ зависит от $\alpha^{\prime}$ 


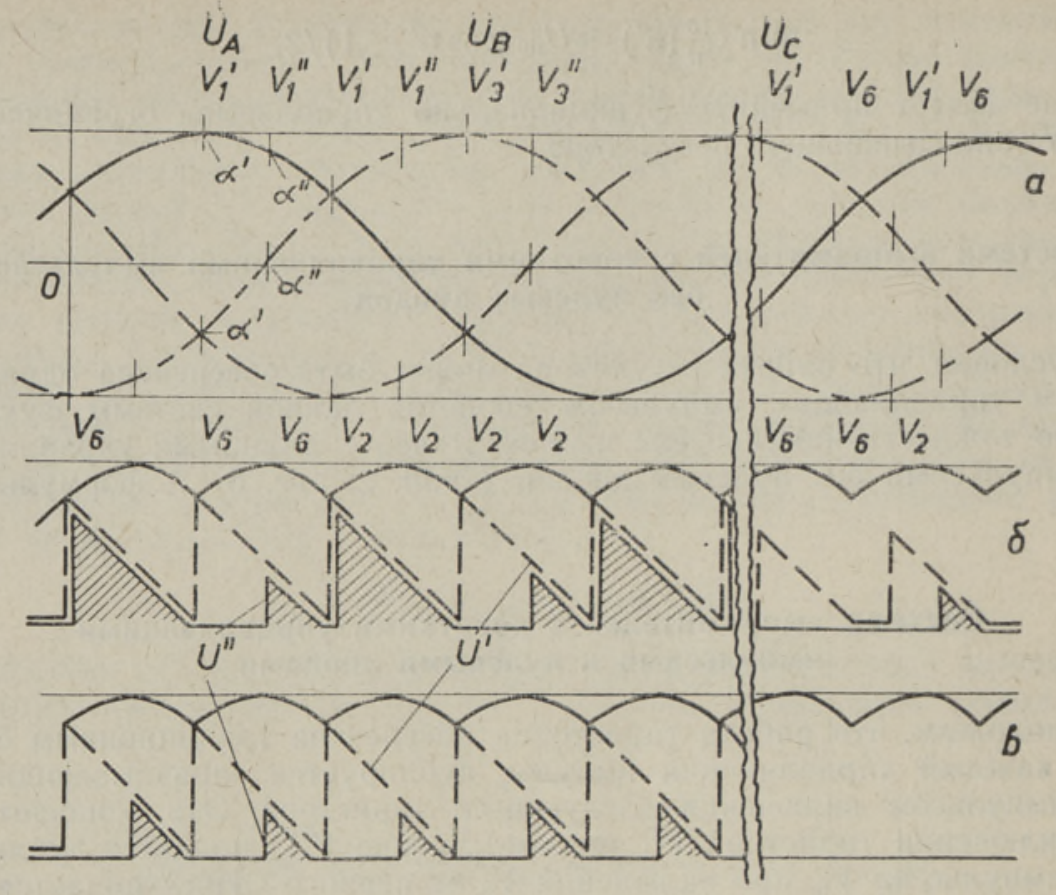

Рис. 8.

скачкообразно. При бесконечно малом изменении $\alpha^{\prime}$ в окрестности $\pi / 3$ происходит изменение выходного напряжения на следующую величину:

$$
\Delta U_{d}^{\prime \prime}=-\frac{U_{d 0}}{4}\left[1+\cos \alpha^{\prime \prime}-\sqrt{3} \sin \alpha^{\prime \prime}\right],
$$

что не является допустимым. Скачок имеет максимальное значение

$$
\max \Delta U_{d}^{\prime \prime}=U_{d 0} / 4 \quad \text { при } \quad \alpha^{\prime \prime}=2 / 3 \pi .
$$

При постепенном увеличении угла управления запаздывающего регулятора он вновь отпирается за счет того, что образуется цепь тока через вентиль общей группы следующего канала и вентиль запаздывающей группы. Для этого $V_{2}$ должен отпираться ранее $V_{1}^{\prime \prime}$, что возможно при условиях $\alpha^{\prime \prime}>2 / 3 \pi$ и $\Delta_{\alpha} \geqslant / 3$. Названное обусловливает также скачкообразное запирание (отпирание) запаздывающего регулятора. Видно, что данная система пригодна для работы лишь в ограниченном случае $\left(\boldsymbol{\alpha}^{\prime}, \boldsymbol{\alpha}^{\prime \prime}\right)<\pi / 3$, что вряд ли устраивает с практической точки зрения. Однако существует возможность применения и коротких управляющих импульсов в данной системе, что требует усложнения алгоритма управления общей тиристорной группы. Неоднозначность устраняется подачей дополнительных управляющих импульсов на тиристоры общей группы с фазовым сдвигом $\alpha_{\text {opr }}=\pi / 3$. Это показывает, что для прерывистого режима в любом из регуляторов общая группа представляется неуправляемой. Для работы в непрерывном режиме сохраняются свойства управляемости с вытекающим из этого улучшением спектрального состава выходного тока. Точнее, опережающий регулятор сохраняет свойство 6-пульсности, а запаздывающий регулятор является в этом диапазоне также 6-пульсным, однако с несимметрией управления по раздельным группам вентилей. Это свойство сохраняется в диапазоне регули- 
рования $0,5 U_{d 0}<U_{d}<U_{d 0}$, что является предпочтительным с точки зрения коэффициента мощности и к чему стремятся при разработке реальных систем электропитания.

\section{Система выпрямителей с короткими управляющими импульсами и нулевыми диодами при ограничении диапазона управления общих тиристоров}

Опережающй регулятор имеет в первой зоне работы при $0<\alpha^{\prime}<\pi / 3$ свойства обычного 6-пульсного моста. Регулировочная характеристика описывается формулой (3). Во второй зоне при $\pi / 3<\alpha^{\prime}<2 / 3 \pi$ все четные тиристоры работают с углом ограничения $\alpha_{\text {opr }}=\pi / 3$, а все нечетные тиристоры - с истинным углом управления $\alpha^{\prime}$. Пределы интегрирования для (2) составляют:

$$
\alpha_{i H}=\alpha^{\prime} ; \quad \alpha_{i k}=2 / 3 \pi, \quad \alpha_{j H}=2 / 3 \pi, \quad \alpha_{j k}=\pi,
$$

где $U^{\prime}{ }_{d}$ выражается формулой (10) при $\alpha \in\left\{\alpha^{\prime}\right\}$. В третьей зоне работы при $2 / 3 \pi<\alpha^{\prime}<\pi$ опережающий регулятор приобретает свойства обычного полууправляемого моста, для которого, как известно, $U^{\prime}{ }_{d}$ выражается формулой (7) при $\alpha \in\left\{\alpha^{\prime}\right\}$.

Расчетная и экспериментальная регулировочные характеристики опережающего регулятора приведены на рис. 9 .

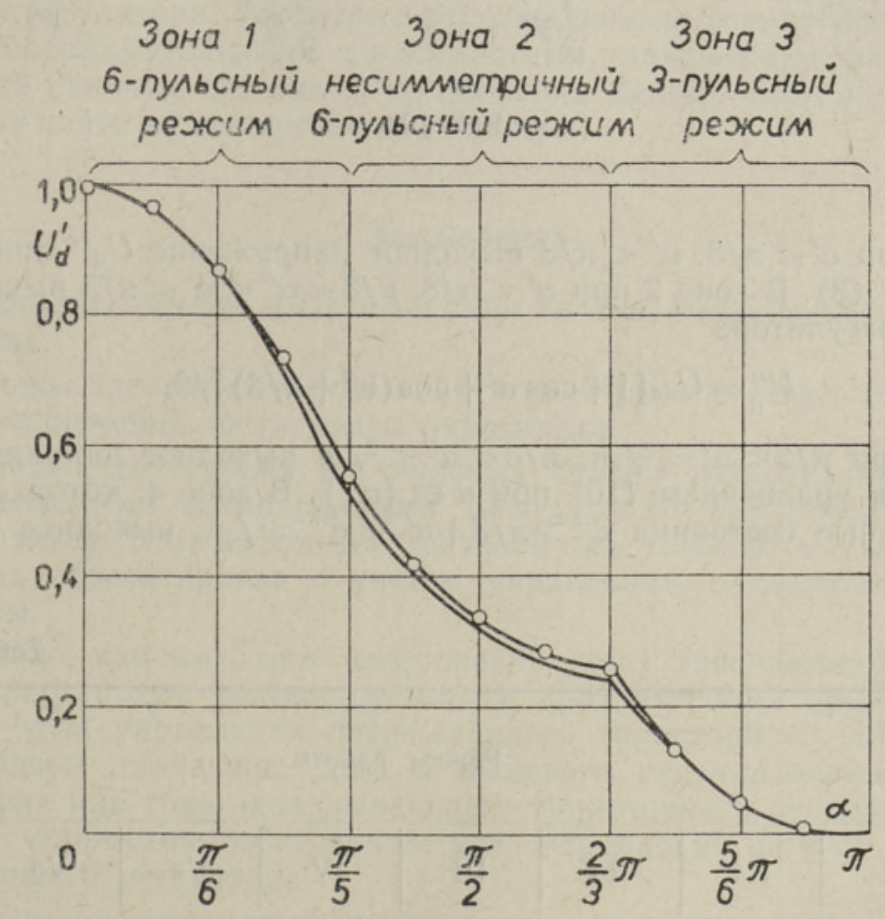

Рнс. 9.

Запаздывающий регулятор. Исходные данные для анализа по (2) сосредоточены в табл. 3. Регулировочные характеристики запаздываю- 
щего регулятора описываются по зонам работы, показанным на рис. 10 следующим образом.

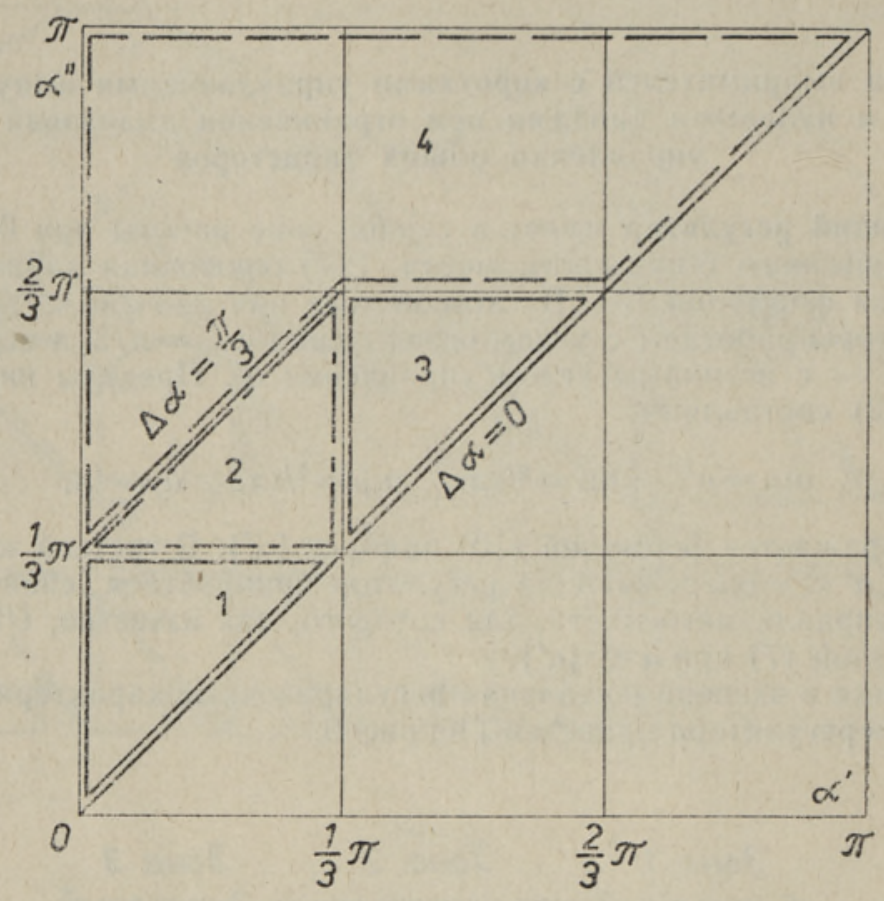

Рнс. 10.

В зоне 1 при $\alpha^{\prime}<\pi / 3, \alpha^{\prime \prime}<\pi / 3$ выходное напряжение $U_{d}^{\prime \prime}$ описывается уравнением (8). В зоне 2 при $\alpha^{\prime}<\pi / 3, \pi / 3<\alpha^{\prime \prime}<\alpha^{\prime}<\pi / 3$ выходное напряжение регулятора

$$
U_{d}^{\prime \prime}=U_{d 0}\left[1+\cos \alpha^{\prime}+\cos \left(\alpha^{\prime \prime}+\pi / 3\right)\right] / 2 .
$$

В зоне 3 при $\pi / 3<\alpha^{\prime}<2 / 3 \pi, \pi / 3<\alpha^{\prime \prime}<2 / 3 \pi$ выходное напряжение $U_{d}^{\prime \prime}$ описывается уравнением (10) при $\alpha \in\left\{\alpha^{\prime \prime}\right\}$. В зоне 4 , которая охватывает остальные состояния $\alpha^{\prime \prime}>\pi / 3+\alpha^{\prime} \bigvee \alpha^{\prime \prime}>^{2} / 3 \pi$, выходное напряже-

Таблица 3

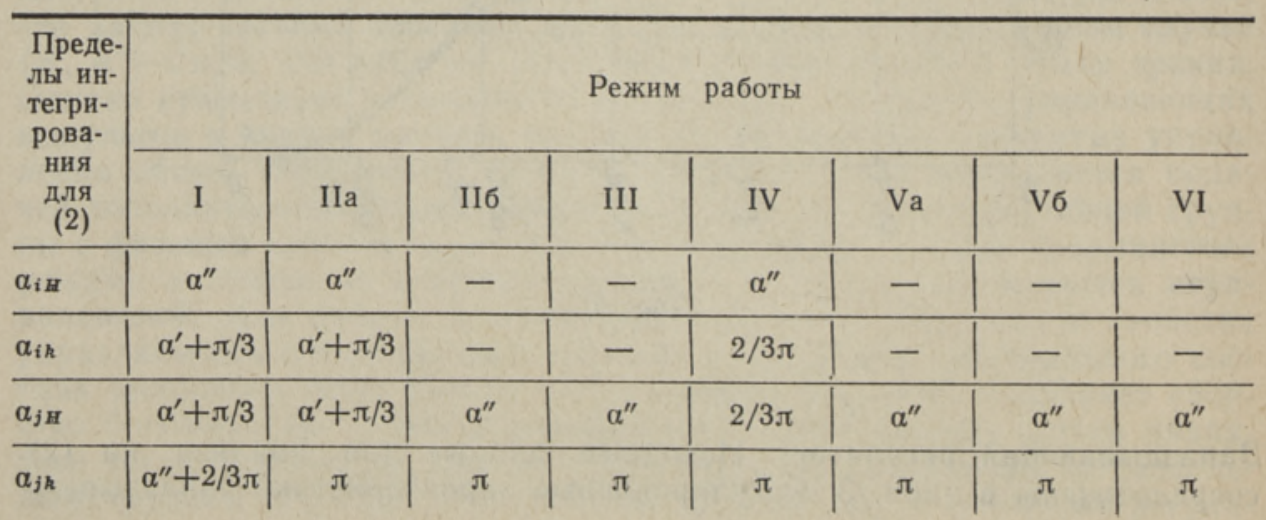




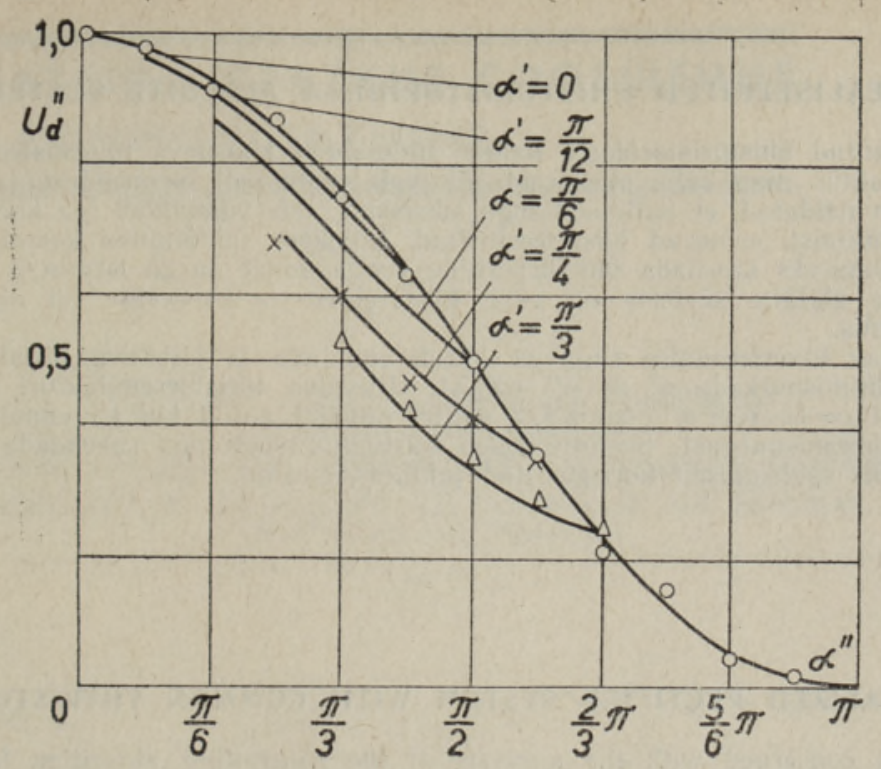

Рнс. 11.

ние описывается уравнением (7) при $\alpha \in\left\{\alpha^{\prime \prime}\right\}$. В зонах 3 и 4 выходное напряжение $U_{d}^{\prime \prime} \neq f\left(\alpha^{\prime}\right)$ инвариантно возмущающему действию опережающего регулятора. Расчетные регулировочные характеристики совместно с экспериментальными зависимостями приведены на рис. 11. Ввиду отсутствия скачков выходного напряжения данная система выпрямителей может найти практическое применение.

\section{Заключение}

1 Разработаны основы классификации систем выпрямителей на общих тиристорах.

2. Получены алгоритмы управления тиристорами в этих системах и выяснены ограничения достигаемых параметров.

3. Показано, что система без нулевых диодов обладает наибольшими функциональными возможностями инвариантно характеру управляющего импульса. Этот вывод действителен при условии, что мостовой выпрямитель спроектирован с учетом управления одиночным коротким импульсом.

4. Показано, что выходное напряжение (ток) запаздывающего тиристорного регулятора, рекомендуемого к практическому применению, зависит от угла управления опережающего тиристорного регулятора во всем рабочем диапазоне. Для автономного регулирования выходного напряжения или тока запаздывающего тиристорного регулятора разумно ввести коррекцию собственного угла управления по углу управления опережающего регулятора.

\section{ЛИТЕРА Т У РА}

1. Тамкиви П., Томсон Т. Изв. АН ЭССР. Физ. Матем., 31, № 4, 454-457 (1982).

2. Калмо Т. Я., Тамкиви П. Н., Томсон Т. И. Источниқ питания. Авт. свид. СССР № 1008865. Бюл. нзобр., № 13 (1983). 


\section{-IDEALISEERITUD OHISTURISTORIDEGA ALALDITE SUSTEEM}

Artiklis on antud ühistüristoridega, kahest sildalaldist koosneva toitesüsteemi juhtimisalgoritmi analüüs ning selle piirangud sõltuvalt nulldioodi olemasolust ja juhtimpulsi pikkusest. On näidatud, et nulldioodidega süsteemis, mis võimaldab iga koormuse selektiivset väljalülimist, esinevad hüstereesinähud. Lühikese juhtimpulsi korral vōib hüstereesi kõrvaldamiseks kasutada ühistüristoride sundavamist nurga $\omega t=\pi / 3$ juures. Ilma nulldioodideta alaldite süsteem on vaba hüstereesist ja kasutatav nii alaldi- kui ka invertorirežiimis.

Saavutatav inverteerimise sügavus sõltub ennetuva ja hilistuva türistorregulaatori vastavate juhtimisnurkade $\alpha^{\prime}$ ja $\alpha^{\prime \prime}$ vahest. Hilistuva türistorregulaatori väljundpinge keskväärtus $U_{d}=U_{d 0}\left(\cos \alpha^{\prime \prime}+\cos \alpha^{\prime}\right) / 2$ sõltub niihästi antud kui ka ennetuva türistorregulaatori juhtimisnurgast. Seetōttu tuleb käsitletud süsteemis rakendada autonoomse töö tagamiseks spetsiaalselt korrigeeritud juhtimissüsteemi.

\section{T. TOMSON}

\section{IDEALIZED RECTIFIER SYSTEM WITH COMMON THYRISTORS}

The paper is concerned with the analysis of the controlling algorithm for a supply system consisting of two bridge rectifiers with common thyristors and its limitations, depending on the presence of the free-wheel diode and the length of the control pulse. It is shown that the phenomenon of hysteresis occurs in the free-wheel diode system enabling the selective turn-off of the load. By using the short control pulse, the hysteresis can be eliminated by the forced commutation of common thyristors at the angle of $\omega t=\pi / 3$. The rectifier system without free-wheel diodes is free from hysteresis and can be used both for the rectifier and inverter mode.

The obtained degree of inversion depends on the difference between the control angle of lead $\alpha^{\prime}$ and the control angle of delay $\alpha^{\prime \prime}$ on the thyristor regulator. The mean value of the output voltage over the delay thyristor regulator $U_{d}=U_{d 0}\left(\cos \alpha^{\prime \prime}+\right.$ $\left.+\cos \alpha^{\prime}\right) / 2$ is dependent both on the given control angle and the angle of lead of the thyristor regulator. Therefore a specially corrected control system must be used in the discussed system to provide independent operation. 